\title{
Multiple Large Cysts Arising from Nevus Comedonicus
}

\author{
Hii-Sun Jeong ${ }^{1}$, Hye-Kyung Lee ${ }^{1}$, Seung-Hyun Lee ${ }^{1}$, Hyoung-Suk Kim ${ }^{1}$, Sang-Yeop Yi ${ }^{2}$ \\ Departments of ${ }^{1}$ Plastic and Reconstructive Surgery, and ${ }^{2}$ Pathology, Myongji Hospital, Kwandong University College of Medicine, \\ Goyang, Korea
}

\begin{abstract}
Nevus comedonicus is a type of hamartoma that arises from a developmental anomaly of the mesodermal part of the pilosebaceous gland. In most cases of nevus comedonicus, an acnelike skin condition develops. Repeated inflammation can cause a morphological change to the cyst, papule, to abscess. We experienced a case of congenital nevus comedonicus, which led to the formation of large multiple cysts. A 50-year-old man was referred with a $12.5 \times 10 \mathrm{~cm}$ lobulated mass on the posterior neck and upper back. The patient had a widespread presence of nevus comedonicus in the region ranging from the right superior chest to the posterior neck. The patient had a 30-year history of six prior excisions. A magnetic resonance imaging review led to a diagnosis of nevus comedonicus. Surgical treatment consisted of excision of the mass and wide excision for the patch type of nevus comedonicus around the neck. On histopathology, multiple masses were diagnosed as typical cysts containing keratinized tissue. The diffuse comedone lesions were diagnosed as nevus comedonicus. This case shows that large, multiple cysts can occur as a long-term complication of nevus comedonicus, and also highlights the importance of radical resection to prevent its further invagination.
\end{abstract}

Keywords Cysts / Sebaceous of Jadassohn nevus / Nevus epidermal cyst
Correspondence: Hii-Sun Jeong Department of Plastic and

Reconstructive Surgery, Myongji Hospital, Kwandong University College of Medicine, 55 Hwasu-ro 14 beon-gil, Deogyang-gu, Goyang 412-826, Korea

Tel: $+82-31-810-6830$

Fax: +82-31-969-6837

E-mail: hiisunj@gmail.com

This article was presented as a poster at the 68th Congress of the Korean Society of Plastic and Reconstructive Surgeons on November 4-7, 2010 in Seoul, Korea.

No potential conflict of interest relevant to this article was reported.

Received: 19 Aug 2011 • Revised: 26 Sep 2011 • Accepted: 6 Oct 2011

pISSN: 2234-6163 • elSSN: 2234-6171 • http://dx.doi.org/10.5999/aps.2012.39.1.63 • Arch Plast Surg 2012;39:63-66

\section{INTRODUCTION}

Nevus comedonicus (NC) is a type of hamartoma that occurs around the pilosebaceous gland. It was first reported in 1895 by Koffmann [1]. The incidence of NC is estimated at 1/45,000 [2], with no sex-related differences $[2,3]$. NC principally affects the face, neck, upper limbs, and chest at birth, during infancy, and/ or childhood. Although there are generally no clinical symptoms of NC, the histopathological findings of this disease include enlargement of the hair follicles due to epidermal invagination into the dermis. In cases involving frequent periods of inflammation, NC can undergo morphological changes to a cyst, papule, pus- tule, or abscess.

A case of congenital $\mathrm{NC}$ affecting regions from the anterior chest to the posterior neck and involving the formation of large multiple cysts beneath $\mathrm{NC}$ was surgically treated at our hospital. In cases in which long-term inconvenience and aesthetic outcomes are of primary concern and in cases of small-sized lesions, a radical resection could be considered a treatment of choice. Here, we report this case with a review of the literature.

\section{CASE}

A 50-year-old man presented with widespread $\mathrm{NC}$ in the region 


\section{Fig. 1. Appearance of the nevus comedonicus}

(A) Initial appearance: the multiple and huge masses on the posterior neck. (B) Multiple nevus comedonicus with pits. Complete excision was done with the guidance of a lacrimal probe. (C) MRI findings (T2 weighted image): a mixed signal density of cystic content.
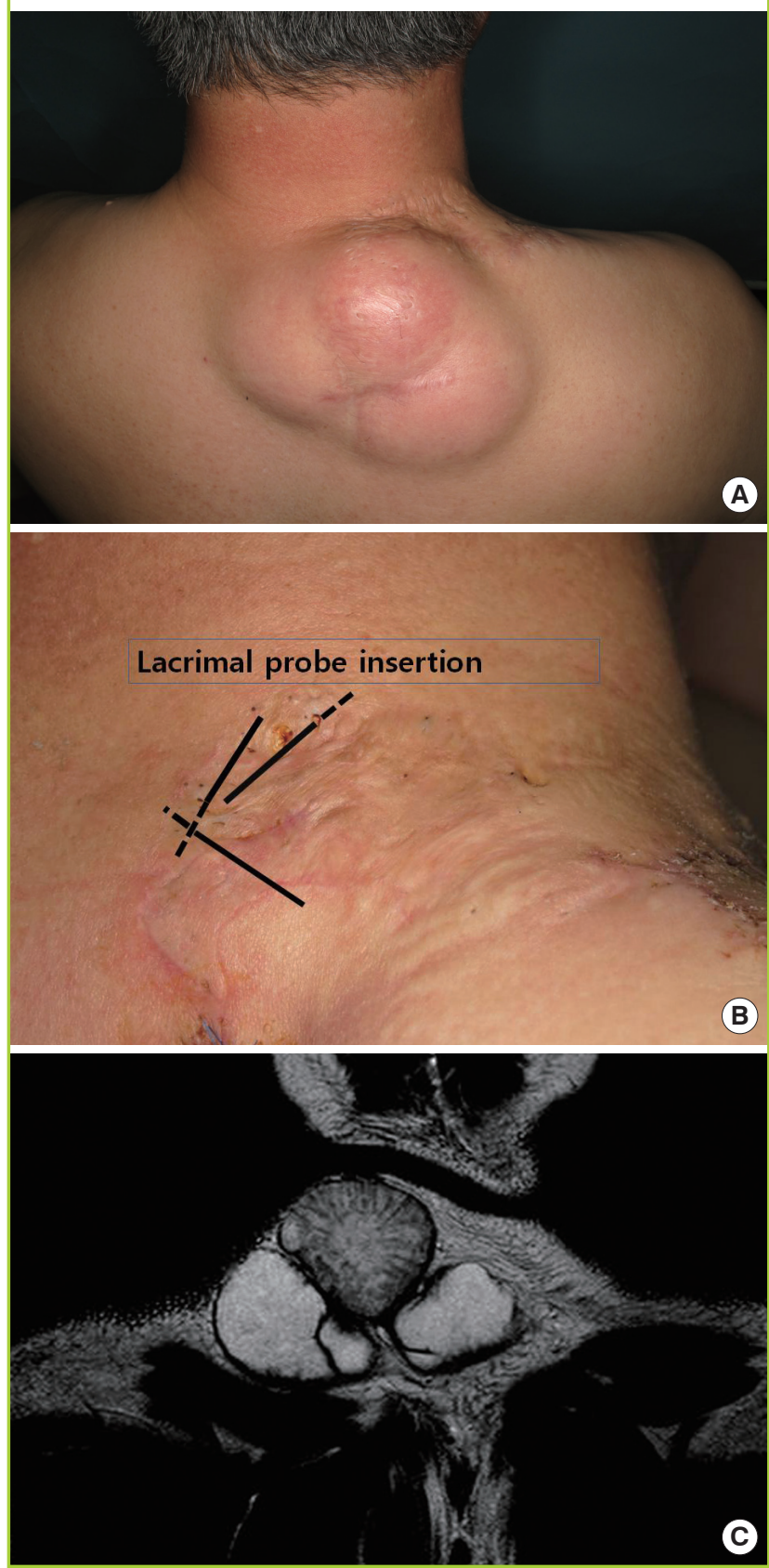

from the right superior chest to the posterior neck and large lump on his posterior neck. This patient had previously undergone a staged excision of cysts and nevus comedonicus six times over the course of 30 years. The patient had undergone an excision for posterior mass at another hospital 8 years previously. The mass was diagnosed as a sebaceous cyst based on histopathology. Thereafter, multiple large cysts grew as a result of cyst recur-

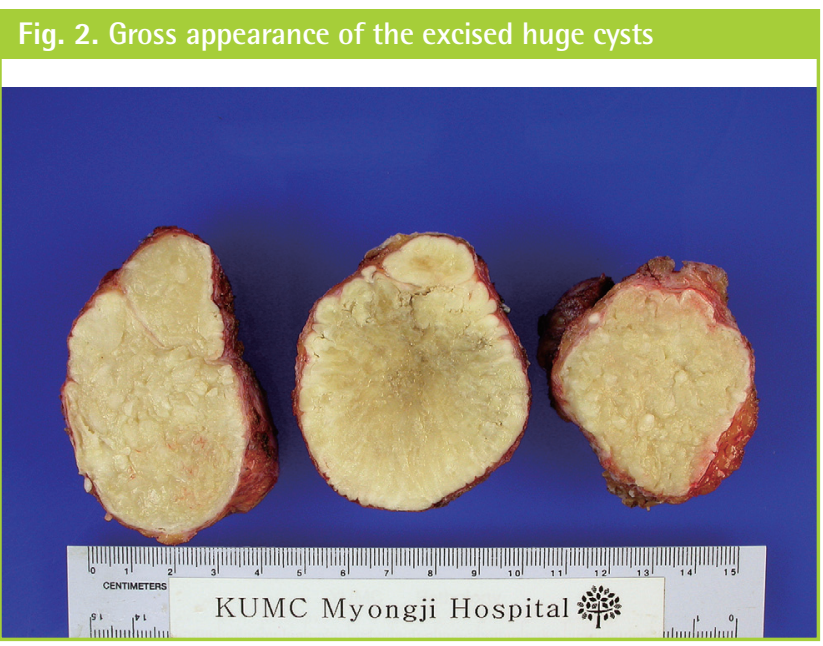

rence. A family history revealed that the patient's brother had NC on his right thigh.

On physical examination, the patient had one cyst on the lower neck and two cysts on the upper back, both of which had pyramidal shapes and dimensions of $12.5 \times 10.0 \mathrm{~cm}$. The mass was soft and fixed, and the adjacent area was somewhat erythematous and mildly warm, leading to suspected inflammation. However, there were no tenderness and no fluctuations. Additionally, results of a complete blood count, urinalysis, and chest radiography were normal. Magnetic resonance imaging (MRI) scans of the neck revealed that the lower mass was shaped typically like a cyst, but the upper mass had a non-typical shape and its center had foreign body components. A total of five masses were detectable (Fig. 1).

Under general anesthesia, the patient underwent a surgical procedure in which the center of the three larger masses was resected using one longitudinal incision. For excising the nevus comedonicus, the incision line was drawn widely by checking the depth and extent of the invaginated orifice with the help of a lacrimal probe. This zig-zag incision line was designed to prevent scar contracture. This procedure prevented the possibility of recurrence and the resection was successfully completed without rupture. Grossly, the surgical samples had a spherical shape and the upper mass was $5.5 \times 5.0 \mathrm{~cm}$ in size (Fig. 2). The two smaller masses were $6.0 \times 5.0 \mathrm{~cm}$ and $6.5 \times 5.0 \mathrm{~cm}$ in size, and were also resected.

Hematoxylin-eosin staining of the three large masses demonstrated that they were typical cysts containing keratinized tissue. In particular, this stain showed that the upper mass with foreign body components detected by MRI of the neck had typical cyst histopathology (Fig. 3). The sites of resection were primarily sutured following drainage, and then healed without complications. Additionally, NC at other sites was also surgically re- 


\section{Fig. 3. Histopathologic findings of cyst tissue}

(A) Histopathologic findings of cysts showed lamellated keratin content was washed out during H\&E preparation (H\&E, $\times 100)$. (B) Histophathologic findings of typical nevus comedonicus showed wide dilation and deep invagination with laminated keratin plugs and perifollicular hyperkeratosis, acanthosis, and papillomatosis $\left(H \& E_{1} \times 40\right)$.
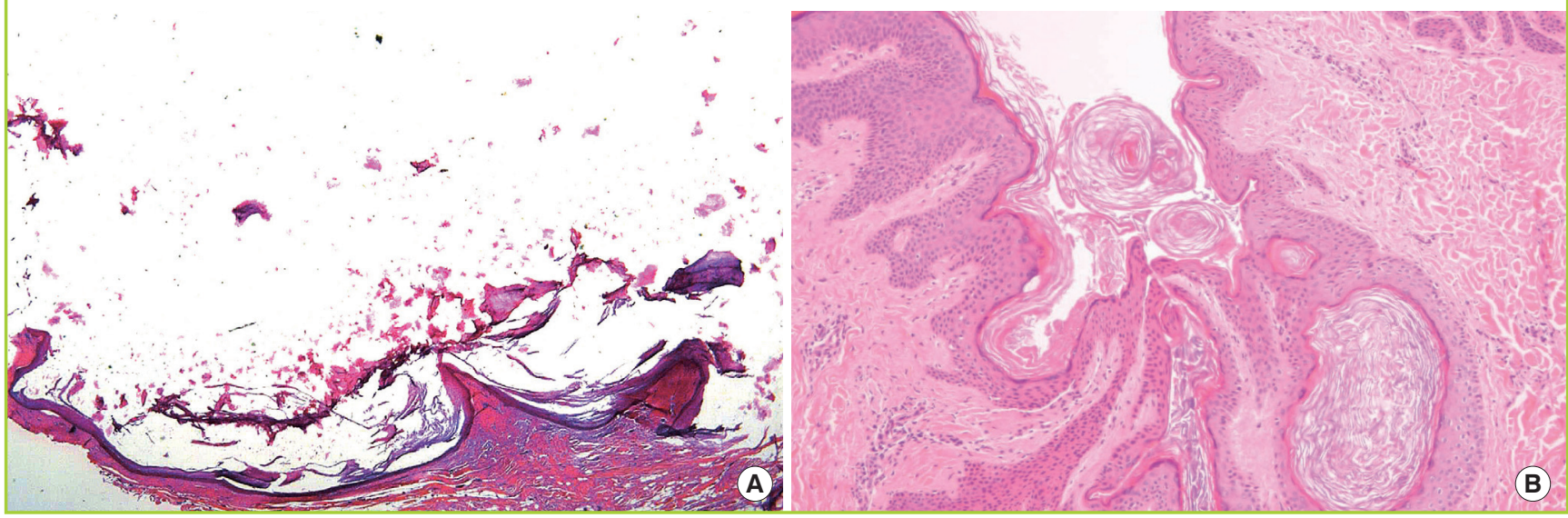

\section{Fig. 4. Postoperative appearance}

Postoperative clinical photo after six months showed a mild depressed and erythematous scar without recurrence.

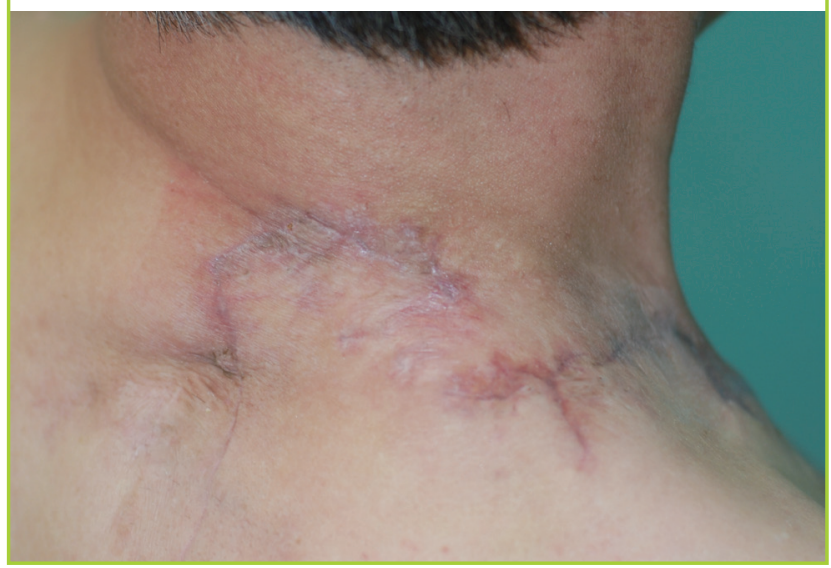

moved. The patient had no recurrences or other complications during a 6-month postoperative follow-up period (Fig. 4).

\section{DISCUSSION}

$\mathrm{NC}$ is classified into two main types based on its clinical characteristics [3]. The first type is non-pyogenic NC with acnelike characteristics. The second type is NC with cysts, papules, pustules, or abscesses that undergo morphological changes. The case described here, which is of the latter type of NC, involved a large cyst that recurred due to previous incomplete surgery. Additionally, the size of this cyst gradually increased due to a lack of sebum secretion due to postoperative invagination of the orifice. Furthermore, expansion of the skin lesions due to increasing size of the cysts results in an elevated risk of infection. Presum- ably, this increased infection risk and frequent periods of inflammation might therefore contribute to the development of large cysts.

Previously published reports of NC cases showed that this condition sometimes presents concurrently with epidermal or skin appendage tumors, such as epidermolytic hyperkeratosis, epidermal nevus, and eccrine nevus [4,5]. Additionally, Engbers [6] reported rare cases in which NC occurred concurrently with other skin lesions or certain types of internal abnormalities or ailments; he termed this condition "NC syndrome". NC syndrome belongs to the large category of epidermal nevus syndromes in which epidermal nevus appears concurrently with abnormalities of other body organs, such as neurological deficits (epilepsy and electrocardiogram abnormalities), skeletal abnormalities (scoliosis and spina bifida), and eye problems (congenital cataracts) [6]. Physical examination and other laboratory test results indicated that the current case was not $\mathrm{NC}$ syndrome.

Histopathologically, NC is characterized by the presence of extensive and deeply stratified keratin in the epidermis. The invaginated epidermis is composed of various types of keratinocytes. It is important for $\mathrm{NC}$ to be clinically differentially diagnosed from acne vulgaris [3]. Histopathological analysis reveals that the skin appendage tumors include dilated pore of Winer, pilar sheath acanthoma, and trichilemmal cyst [7]. In the present case, histopathology showed that the large cyst was typical of those containing keratinized tissue. The histopathological findings in this case were almost identical to those of a sebaceous cyst.

Because of recurrent infections and cosmetic problems, NC poses a great challenge to clinicians, and the only effective treatment method is surgery. It is important for those with NC to 
maintain good hygiene, and antibiotics can also be used as a conservative method of treatment. As a localized treatment, retinoic acid can be used to induce the expansion and differentiation of keratinocytes, which would be effective for the elution of nevus. In addition, ammonium lactate lotion [1] softens the epidermis, thereby promoting the excretion of nevus. Other available treatments include the mechanical removal of nevus, dermabrasion, or laser therapy. However, these methods cannot prevent recurrence of the lesion [3] and surgical removal is the treatment of choice for NC. In cases involving a smaller area, the primary suture should be performed following resection. Cases with larger affected areas require staged excision or skin grafting. In some cases, reconstruction using a tissue expander would be helpful.

Due to a time-dependent increase in the risk of opportunistic infections, NC undergoes morphological changes to cysts, papules, pustules, or abscesses. Non-surgical supplemental treatment methods can be effective to a limited degree. Our case showed that large, multiple cysts could occur as one of the long-term complications of NC, and it also suggests the importance of radical resection for further invagination of the lesion. Besides, it also indicates that $\mathrm{NC}$ should be differentially diagnosed from soft tissue tumors based on the possibility of progressing to large lesions.

In conclusion, the present case highlights the importance of an early radical surgical approach to treat even cases with a small area of nevus.

\section{REFERENCES}

1. Kofmann S. Ein fall von seltener lokalisation und verbreitung von comedonen. Arch Dermatol Syphilis (Wien) 1895;32:177-8.

2. Marcus J, Esterly NB, Bauer BS. Tissue expansion in a patient with extensive nevus comedonicus. Ann Plast Surg 1992;29:362-6.

3. Beck MH, Dave VK. Extensive nevus comedonicus. Arch Dermatol 1980;116:1048-50.

4. Kim SC, Kang WH. Nevus comedonicus associated with epidermal nevus. J Am Acad Dermatol 1989;21:1085-8.

5. Barsky S, Doyle JA, Winkelmann RK. Nevus comedonicus with epidermolytic hyperkeratosis: a report of four cases. Arch Dermatol 1981;117:86-8.

6. Engber PB. The nevus comedonicus syndrome: a case report with emphasis on associated internal manifestations. Int J Dermatol 1978;17:745-9.

7. Kim YJ, Hong CY, Lee JR. Nevus comedonicus with multiple cyst. J Korean Cleft Palate-Craniofac Assoc 2009; 10:135-7. 